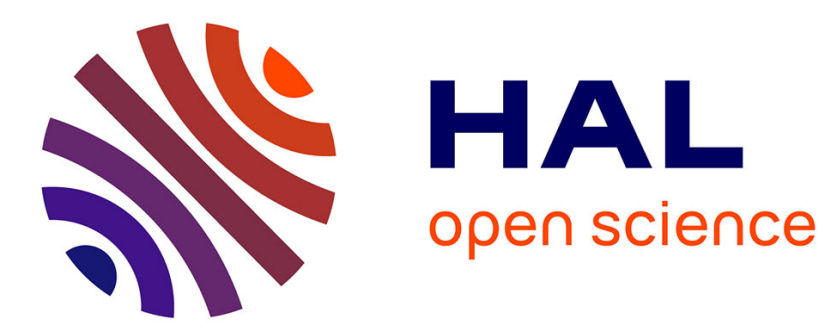

\title{
Questions d'intuition
}

Samir Bajrić

\section{To cite this version:}

Samir Bajrić. Questions d'intuition. Langue française, 2005, 147 (1), pp.7 - 18. 10.3406/lfr.2005.6860 . hal-01619703

\section{HAL Id: hal-01619703 \\ https://u-bourgogne.hal.science/hal-01619703}

Submitted on 24 Nov 2017

HAL is a multi-disciplinary open access archive for the deposit and dissemination of scientific research documents, whether they are published or not. The documents may come from teaching and research institutions in France or abroad, or from public or private research centers.
L'archive ouverte pluridisciplinaire HAL, est destinée au dépôt et à la diffusion de documents scientifiques de niveau recherche, publiés ou non, émanant des établissements d'enseignement et de recherche français ou étrangers, des laboratoires publics ou privés. 


\section{Questions d'intuition}

\section{Samir Bajrić}

\section{Abstract}

Samir Bajric : Linguistic analysis of intuition.

This paper deals with the linguistic analysis of intuition (the immediate understanding of something without conscious reasoning or study). The theoretical framework is Gustave Guillaume's psychomechanics of language and mental approaches in the linguistics. However, the greatest influence of philosophy upon this topic is found in L. Wittgenstein's and H. Bergson's works. This is done by observing a great number of circumstances within the context of the intuitive understanding and statement. Three main issues are discussed : intuition of statement, heunstic intuition and analogical intuition. In cases where the (strictly) grammatical patterns have not been preserved, the intuitive approach has been identified.

\section{Citer ce document / Cite this document :}

Bajrić Samir. Questions d'intuition. In: Langue française, $n^{\circ} 147$, 2005. La langue française au prisme de la psychomécanique du langage. Héritages, hypothèses et controverses. pp. 7-18;

doi : 10.3406/fr.2005.6860

http://www.persee.fr/doc/lfr_0023-8368_2005_num_147_1_6860

Document généré le 13/06/2016 


\section{Samir Bajric}

Université de Paris IV-Sorbonne

\section{Questions d'intuition}

\section{O. BRÈVE INTRODUCTION}

En révisant l'histoire des idées linguistiques du siècle précédent, nous constatons que les sciences du langage se sont approprié un certain nombre de disciplines annexes permettant ainsi l'émergence d'une épistémologie nouvelle, plus " épaisse " et capable de fédérer en un seul faisceau interprétatif les domaines suivants : linguistique informatique (TAL), psycholinguistique, neurolinguistique, philosophie du langage, traductologie, didactologie des langues-cultures, etc. Dans ce contexte formel et temporel, il est possible, quel que soit le domaine retenu, de s'intéresser, $d$ 'une part, à la nature des résultats issus de différentes activités langagières et, d'autre part, aux processus cognitifs qui précèdent ces activités.

La plupart des linguistes, lorsqu'ils tentent $d^{\prime}$ 'appréhender le phénomène langagier, se bornent à décrire le fonctionnement des éléments linguistiques entérinés par l'usage. Ils s'intéressent peu à la composante mentale du langage, celle qui " prépare " et conditionne l'énonciation dans toute sa variété. Cette composante renvoie à l'ensemble des opérations linguistiques accomplies la plupart du temps insciemment (à notre insu) et qui sont, nolens volens, des opérations de haute raison. Ces opérations peuvent être décrites à travers les notions de mentalisme et d'intuition, qui procurent à la linguistique une dimension hautement ontologique et, par conséquent, plus proche de l'immanence de l'être. L'objectif de cette étude est donc de définir l'intuition en linguistique en cherchant du côté de l'abstrait, en étudiant le phénomène langagier "de l'intérieur ".

Toutes les langues possèdent des propriétés abstraites, non visibles de l'extérieur et inaccessibles à l'analyse des formes linguistiques. Ces propriétés relèvent des universaux du langage. L'intuition linguistique constitue également une entité universelle, étant donné qu'elle procède d'un concept (l'intuition au sens philosophique) universalisant et immanent à l'être humain. Et c'est précisément ce lien entre "l'universel " et "l'intérieur " qui procure à la présente étude sa raison d'être. Dans le cas de l'intuition, qui constitue l'objet d'étude de la linguistique mentaliste, ce lien doit être particulièrement renforcé. Selon Guillaume, «l'univers avec lequel nous entrons en contact est un univers intérieur : l'univers du 
pensable, celui que constituent en nous nos représentations. Cette intériorité ajoute beaucoup aux difficultés de l'observation : il est difficile, en effet, que nous saisissions avec exactitude ce qui se passe au fond de nous-mêmes. La difficulté vient, pour une grande part, de ce que nous arrivons toujours en retard pour observer. Voulons-nous observer un acte de langage, nous ne pouvons le faire qu'une fois cet acte accompli, ce qui revient à dire que nous n'avons pas observé l'acte, le procès lui-même, mais seulement son résultat. C'est insuffisant. »'

Force est de constater que la difficulté d'observation devient plus grande encore lorsqu'il s'agit d'examiner les faits à partir de leur résultat. Nombre de sciences (dont certaines disciplines au sein même des sciences du langage, comme le TAL, par exemple) s'interrogent sur des éléments dont elles ignorent (et parfois appréhendent) le résultat final, ce qui, au demeurant, constitue le cas contraire par rapport à la pensée de Guillaume, citée précédemment. Dans le cas présent, le linguiste est enclin à retrouver, dans les faits de langage et/ou dans les faits de langue, le point de départ, ce processus qui permet de "traduire en dicibilité des mécanismes dont nous portons en nous, préalablement, la visibilité. ${ }^{2}$. La dichotomie dicibilité/visibilité appelle des considérations mentalistes favorisant l'examen des opérations pré-énonciatives, celles qui conditionnent le résultat d'un acte de langage. Il semble donc judicieux de s'intéresser aux acquis théoriques du mentalisme en linguistique avant même de proposer une définition de l'intuition.

\section{Le MeNTALISME EN LINGUISTIQUE}

Il convient d'emblée de préciser les enjeux épistémologiques de cette recherche. Le mentalisme ne constitue pas une nouveauté conceptuelle dans les sciences du langage. Certains courants linguistiques mettent à profit, depuis plusieurs décennies, une observation du langage "de l'intérieur ». D'autres se limitent essentiellement aux manifestations post-énonciatives. D'où l'idée de songer à au moins deux types d'épistémologie linguistique :

1) les théories linguistiques d'inspiration (ouvertement) mentaliste, dans lesquelles le phénomène langagier n'est envisagé qu'intrinsèquement, $c^{\prime}$ est-à-dire dans ses structures sous-jacentes et non observables de l'extérieur. Tel est le cas, nous le savons, de la psychosystématique du langage de Gustave Guillaume. La psychosystématique propose un modèle interprétatif dans lequel le mentalisme apparaît sous la forme la plus transparente. « De toutes les relations et corrélations intervenantes dans le langage et dans la science observatrice du langage, la plus importante - et la plus négligée (celle dont il est le moins tenu compte) - est la relation du physique et du mental. " ${ }^{3}$. Cette disposition théorique, établie par Guillaume, consolide notre conviction que l'intuition, définie provisoirement comme la représentation directe de la relation entre le physique et le mental, habite les structures langagières.

1. Guillaume, 1973, pp. 37-38.

2. Guillaume, ibidem, p. 38.

3. Guillaume, ibidem, p. 121. 
2) les théories où la dimension proprement mentaliste émerge de façon plus discrète. Tel est le cas, par exemple, de la syntaxe structurale de Lucien Tesnière qui véhicule une conception mentaliste non avouée, notamment dans ses notions de connexion, de verbe-régissant et de translation. Nous partons du principe que cette théorie, contrairement à de nombreuses idées reçues, n'est pas réductible à son analyse "post-énonciative " du langage et des langues. Cette conviction est exprimée par Tesnière même, notamment dans son attachement pour la méthode introspective. "La méthode introspective est également objective parce qu'elle porte sur des faits. Certes, ces faits sont abstraits. Mais il n'existe pas que des faits concrets. On peut même dire qu'en syntaxe tous les faits sont abstraits, puisque après tout ce n'est jamais que leur marquant qui est concret. Or il n'y a guère $\mathrm{d}$ 'autre méthode pour atteindre les faits syntaxiques purement abstraits que celle de l'analyse interne. " 4 .

Interne, intérieur, intériorité, ... ces termes confirment que les deux théories convergent et se rassemblent autour du même objectif. Ainsi le concept de " mentalisme " permet-il de concevoir la langue comme un " ouvrage construit en pensée ", opposé à celui de physisme qui identifie à l'intérieur de la langue un " ouvrage construit en signes" (Guillaume, 1964, p. 33). Dans ce contexte, il semble néanmoins plus aisé d'étudier la véritable nature du langage « en se portant du côté de l'abstrait qu'en se portant du côté du concret. " (Guillaume, 1985, Leçons, Tome 10, p. 223).

On l'aura bien compris, la conception du langage de Tesnière comporte également une composante mentaliste ou, du moins, une préoccupation d'ordre noologique non réductible à l'analyse purement syntaxique de la phrase. Dans son œuvre capitale (1959), l'auteur évoque plusieurs notions qui évoquent l'intériorité du langage. Nous en retiendrons quelques-unes : "faits de langue saisissables" (p. 13), " superstructure sémantique » (p. 18), « quadrature du cercle du langage » (p. 21), "en puissance » (p. 382), " formes complexes de la pensée » (p. 383), etc. Il considère que la connexion même, concept central de sa théorie, " est indispensable à l'expression de la pensée » (p. 12), qu'il ne faut sous aucun prétexte perdre de vue l'importance de l'innere Sprachform, "qui est par définition intérieure ", parce qu'elle ne se présente pas " sous une forme matérielle » (p. 13), que la méthode introspective « fait appel à l'intuition » (p. 38), et même que la notion de translation doit être " cherchée et devinée par intuition" (p. 381). Ces citations sont de nature à justifier un rapprochement entre la syntaxe structurale et la dimension mentaliste du langage.

Si la langue, l'une des entités de la dichotomie langue/discours, correspond à la position centrale du phénomène langagier (linguistique de position de G. Guillaume), le mentalisme de Guillaume est un mentalisme centripète, dans la mesure où il se rapproche de ce centre. À l'inverse, le mentalisme de Tesnière est un mentalisme centrifuge, dans la mesure où il s'éloigne de ce centre. Le rapprochement de l'un et l'éloignement de l'autre constituent deux axes d'études distincts, mais provenant d'une source commune.

Cette interaction, non équitable, entre un "mentalisme structural » et un "structuralisme mental ", fut appréhendée dans un excellent article publié par C. Puech et D. Savatovsky en 1982 et intitulé Structuralisme et/ou Mentalisme?

4. Tesnière, 1982 (1959), p. 38. 


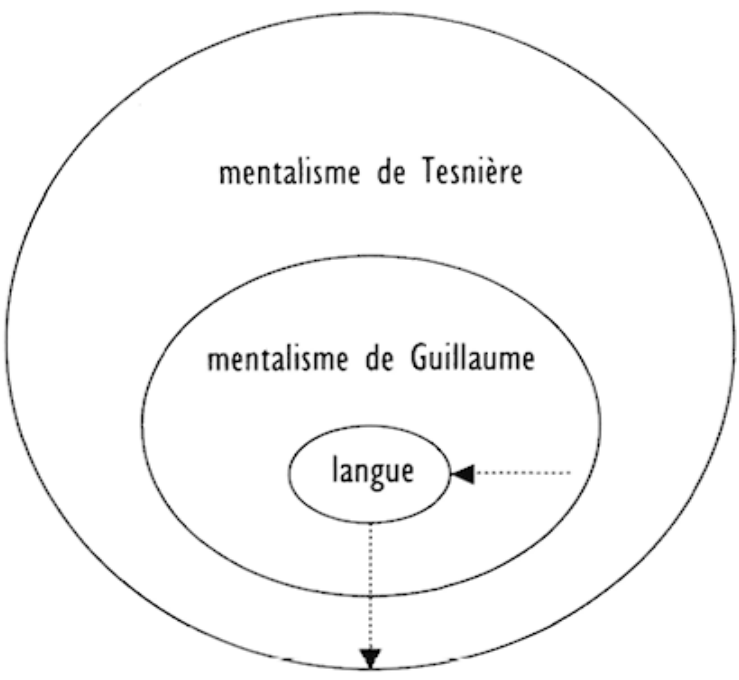

G. Guillaume ${ }^{5}$. C'est également de cette rencontre que naît l'idée d'un comprendre au sens guillaumien (" un comprendre qui conduit à un voir supérieur"; " comprendre, c'est théoriser »), ou encore celle qui renvoie à la dichotomie tesnièrienne : parler une langue/comprendre une langue. Tesnière estime, en effet, que " parler une langue, c'est en transformer l'ordre structural en ordre linéaire, et inversement que comprendre une langue, $c^{\prime}$ est en transformer l'ordre linéaire en ordre structural. ${ }^{6}$.

Cette opération, mentale par excellence, n'est ni simple ni anodine. Et pourtant, elle est inconsciente. Nous parlons et nous comprenons une langue (" notre " langue) de manière "fatale ", dans la mesure où notre faculté langagière relève directement de la nature humaine. Elle constitue l'immanence même de l'être humain. C'est bien cet aspect-là qui introduit la composante mentaliste dans les études linguistiques. Les acquis communs de la psychosystématique du langage et de la syntaxe structurale traduisent l'écart que l'on reconnaît entre méthode introspective et méthode expérimentale. Nous reprenons un schéma de Guillaume (1973, p. 41) que nous élargissons quelque peu pour les besoins de notre analyse :

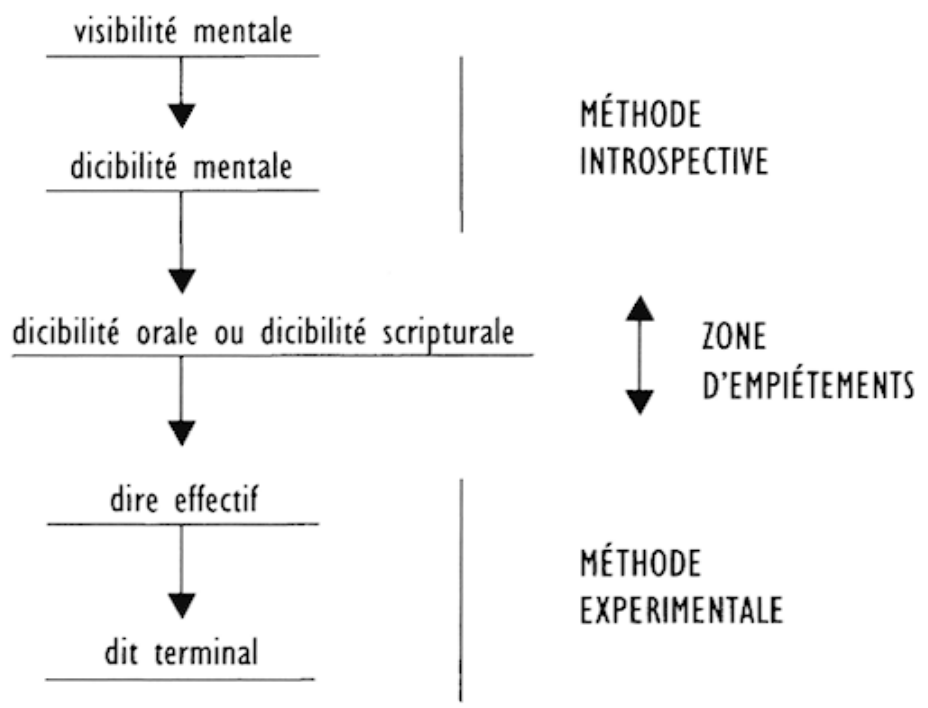

Ainsi apparaissent trois domaines où les faits de langage et/ou les faits de langue deviennent observables. En allant vers l'amont des processus psychiques, l'analyse linguistique balise un premier domaine incluant le dit terminal et le dire effectif. Ce domaine ressortit à la méthode expérimentale, celle qui rend compte des résultats énonciatifs. Un deuxième domaine se construit autour de la dicibilité orale ou la dicibilité scripturale. C'est précisément là, à l'endroit où se croisent le dire et le dicible, que l'analyse linguistique s'intériorise et nous mène "à la visibilité des actes mentaux. "7. Le troisième domaine, enfin, introduit le plus haut degré de

5. D'ailleurs, il suffit de relire les écrits de Guillaume (Leçons et PLT) pour se rendre compte qu'il est le premier à revendiquer cette double appartenance aux sciences du langage : "Tout de même, comme linguiste, comme structuraliste, comme psycho-systématicien du langage, comme observateur de la psycho-sémiologie, je dispose du meilleur document que l'on ait pour l'étude de l'intuition et de cette mécanique très fine, dont nous avons l'instinct, que j'appelle mécanique intuitionnelle et qui est l'opérateur de la structure des langues, laquelle est un miroir fidèle de son activité. » (PLT, p. 44).

6. L. Tesnière, 1982 (1959), p. 19.

7. Guillaume, 1973, p. 42. 
l'intériorité langagière. C'est la perspective mentaliste par excellence, représentée par la méthode introspective. Contrairement aux idées générales, cette méthode ne souffre d'aucune stérilité interprétative. Elle permet de saisir la plus grande variété des faits de langue, notamment des faits syntaxiques (comprendre, porter des jugements d'acceptabilité, innover, etc.). Grâce à la vision mentaliste, il nous est possible d'appréhender les faits syntaxiques avant et après le dit terminal, notamment en les adaptant aux critères de la dichotomie parler une langue/comprendre une langue. Le schéma suivant résumera les modalités de cette relation :

\begin{tabular}{|c|c|}
\hline \multicolumn{2}{|c|}{ FAITS SYNTAXIQUES } \\
\hline parler une langue & comprendre une langue \\
\hline AVANT & APRÈS \\
\hline
\end{tabular}

\section{INTUITION : À PROPOS DU CONCEPT}

Il est indispensable, avant de définir l'intuition en linguistique, de répondre à quelques questions subsidiaires : que signifie le terme "intuition "? L'intuition constitue-t-elle un concept original, autosuffisant et indépendant des systèmes de raisonnement conscients? De quelles disciplines autres que la linguistique relève l'intuition ? Quel est le véritable intérêt de cette étude pour l'analyse linguistique ? Autant de questions préliminaires pour une notion peu connue et difficilement appréhendable.

Le terme intuition vient du latin tueor, intuitio, intueri qui signifie " arrêter son regard sur quelque chose ", "voir à l'intérieur ". L'adjonction du préfixe in précise que cette observation pénètre directement à l'intérieur de la chose. À partir de ce constat, la porte exégétique est ouverte à une profusion de termes scientifiquement plutôt obscurs mais non moins pertinents : "l'eurêka " d'Archimède, "l'illumination " de Descartes, "l'inspiration " des poètes, "la vision de Dieu " des théologiens, "le sixième sens " des parapsychologues, "l'insight " ("images intérieures soudaines " ou " discernement ») du philosophe Konrad Lorenz, "la métaphysique " et, enfin et surtout, "le sentiment linguistique » des locuteurs confirmés (l'exégèse qui consiste à dire : "Je ne sais pas pourquoi, mais je "sens" que tel énoncé n'est pas conforme au système de ma langue "), etc.

Nos dictionnaires usuels définissent l'intuition différemment :

- « une forme de connaissance immédiate qui ne recourt pas au raisonnement ", - « un savoir instantané sans interposition de signes ni de procédés expérimentaux ou déductifs",

- " un acte simple par lequel on se transporte à l'intérieur d'un objet pour coïncider avec ce qu'il a d'unique et d'inexprimable",

- " la conception d'un esprit pur et attentif, conception si facile et si distincte qu'aucun doute ne reste sur ce que nous comprenons ", etc.

D'une manière générale, l'intuition ressortit à la phénoménologie. À ce titre, de nombreuses disciplines se penchent sur le phénomène qu'elle constitue, à commencer par la psychologie empirique, dont le sens même est véhiculé par les expressions suivantes : avoir une intuition, avoir de l'intuition, avoir l'intuition de ce qui va se passer, d'un danger, comprendre qch. ou qqn. d'un seul coup et sans effort, anticiper 
sur qch. ${ }^{8}$, appréhender le monde hors de toute loi logique, etc. En parapsychologie, on l'a dit, l'intuition est considérée comme notre "sixième sens", une façon, affirme-t-on, de "savoir ce que nous ne savons pas". Le domaine des sciences "pures " (par exemple, la physique, l'astrophysique, etc.), n'en est pas plus épargné. Ainsi croiton, pour paraphraser A. Einstein, que la science ne repose ni sur une intuition ni sur une simple déduction logique, mais sur un dialogue subtil entre les deux ${ }^{9}$. La théorie de la relativité, par exemple, est une hypothèse qui a surgi, semble-t-il, de l'imagination d'Einstein.

En philosophie, enfin, on parle d'intuitionnisme, plus précisément de l'intuitionnisme bergsonien. L'intuition joue un rôle essentiel dans la connaissance, dans nos structures cognitives et, plus généralement encore, dans la cognition, définie comme " la faculté de connaître ". ${ }^{10}$ C'est un phénomène purement mental et il $\mathrm{s}^{\prime}$ agit de l'exploiter scientifiquement comme on exploite par exemple le langage. On peut opposer la notion d'intentionnalité (John R. Searle) à la notion d'intuitionnisme. L'intentionnalité est définie comme la conceptualisation de la conscience, c'est-à-dire de nos états intentionnels (doute, inquiétude, intention, imagination, chagrin, colère, etc.). L'intuitionnisme est une prise en considération par laquelle nous rendons inconscientes toutes sortes de connaissances. Pour savoir par quels paradigmes nous accédons à ces opérations créatrices, qui évoluent par ruptures sporadiques et s'opposent ainsi au raisonnement cumulatif, "il devient à peu près inévitable de s'interroger sur la nature de la connaissance et, par conséquent, sur celle de ce qu'il est convenu de nommer l'intellect ou l'esprit. " ${ }^{11}$.

\section{L'INTUITION EN LINGUISTIQUE}

Dans son célèbre ouvrage intitulé La modularité de l'esprit, J. Fodor explique, en quelques phrases, "comment cet ouvrage a vu le jour. " Nous extrayons de ce petit texte ce qui nous semble pouvoir introduire notre étude de l'intuition en linguistique :

Un jour - cela devait se passer il y a environ cinq ans -, mon ami, collègue et parfois co-auteur Merrill Garrett fit la remarque la plus profonde que j'ai jamais entendue sur les mécanismes psychologiques impliqués dans la perception de la parole. « Ce qu'il ne faut jamais oublier ", a dit Merrill, "c'est qu'il s'agit pour l'essentiel d'un réflexe ". Cet ouvrage est en fait une réflexion poussée à partir de l'intuition de Merrill. ${ }^{12}$

En linguistique, l'intuition appartient à un vaste domaine où s'entremêlent de façon générale l'énonciation, l'acquisition des langues, l'acceptabilité grammaticale d'un énoncé, le bilinguisme, les problèmes que posent la traduction, la

8. Jules Verne avait anticipé la conquête de la Lune et situé la base de départ en Floride. De même, les grands joueurs d'échecs ont, semble-t-il, une intuition qui pourrait venir d'une mémoire spatiale des structures des parties, etc.

9. Le mathématicien (allemand) Euler disait : «La science, c'est ce qu'on fait après avoir deviné juste. "

10. J.F. Dortier, 2004, p. 10.

11. E. Gilson, 1969, p. 120.

12. J. Fodor, 2004 (1983), p. 7. 
compréhension des phrases, etc. Une idée de Wittgenstein, renvoyant aux caractéristiques mentales du sujet parlant, vient à l'appui de la conception philosophique de l'intuition: "Je parle sans penser à rien. À vrai dire, je pense à quelque chose. À quoi donc ? Eh bien, à ce que je dis. " ${ }^{13}$. Comment peut-on décrire la nature de certaines réactions linguistiques, provoquées par différents stimuli (au sens bloomfieldien), nous permettant d'énoncer des mots et des phrases sans qu'il y ait eu préparation ni structuration quelconques?

D'où vient donc l'idée d'étudier l'intuition en linguistique et, de surcroît, au sein de l'interprétation mentaliste du phénomène langagier ? Nombre de linguistes utilisent les mots intuitif, intuitivement, intuition, soit lorsqu'ils s'intéressent à la définition d'une unité communément admise comme étant problématique, soit lorsqu'ils s'interrogent sur l'acceptabilité grammaticale d'un exemple concret. Ainsi apprend-on, à titre d'illustration, que les notions de mot et de phrase sont des notions " intuitives ", et que le locuteur (naï) approuve ou désapprouve " intuitivement " l'acceptabilité de tel exemple, sans en fournir la moindre explication, sans être en mesure d'argumenter son acceptation ou son refus. Ce problème a été très largement commenté par $D$. Leeman dans un ouvrage consacré à la phrase complexe. Nous en proposons la citation suivante :

La démarche est d'ordre formel, ce qui signifie qu'elle s'appuie sur l'observation du comportement des formes, évalué à l'aune de l'intuition que nous possédons de ce qui peut se dire ; ce jugement ne se confond pas avec le sens attribué aux unités manipulées : par exemple, les deux suites

Ce vase vient de Chine

Ce vase vient de Japon

sont tout aussi interprétables et ont en première approximation le même sens, moyennant la substitution de Japon à Chine; notre sentiment que l'on dit $\mathrm{Ce}$ vase vient $d u$ Japon plutôt que $\mathrm{Ce}$ vase vient de Japon et inversement $\mathrm{Ce}$ vase vient de Chine plutôt que $\mathrm{Ce}$ vase vient de la Chine n'est pas lié à l'appréhension d'une différence sémantique mais au savoir (inconscient) que, dans de tels cas, la seule forme de est appropriée si le nom qui suit est au féminin, inappropriée autrement (...) Le sens qu'on attribue à ces énoncés ne permet pas de prévoir la contrainte - ce dont témoignent les erreurs commises lors de l'apprentissage du français comme langue étrangère. ${ }^{14}$

Cette intuition que nous possédons de ce qui peut se dire, comme le note très justement $D$. Leeman, s'acquiert avec la langue. Elle peut être supérieure aux connaissances du système linguistique, dans la mesure où les jugements prononcés émanent directement de nos structures langagières. Nous examinerons ici quelques exemples. Chacune des phrases peut être observée par un linguiste professionnel ou par ce que les linguistes ont l'habitude d'appeler eux-mêmes un «locuteur naï ${ }^{15}$ :

1) Je n'ai, et c'est regrettable, malheureusement pas réussi à convaincre Pierre. (acceptation)

13. L. Wittgenstein, 1963, p. 55.

14. D. Leeman, 2002, p. 7.

15. Un locuteur naï est un " non-spécialiste, qui n'exerce pas consciemment une activité de réflexion sur la langue. ", M. Yaguello, 1988, p. 156. Nous empruntons les quatre premiers exemples à C. Guimier, 1996, Les adierbes du français, Ophrys. 
Le linguiste note que l'adverbe malheureusement, compte tenu de sa position médiane (entre les deux adverbes de négation ne et pas), ne provoque aucune rupture entre son incidence externe de $2^{\mathrm{e}}$ degré et le sens exprimé par l'incise $e t$ c'est regrettable. Le locuteur naïf, lui, confirme cette acceptation intuitivement, en s'appuyant sur le sens global (sens phrastique), à l'intérieur duquel aucun élément ne lui paraît suspect.

2) Je n'ai pas, et c'est regrettable, malheureusement, réussi à convaincre Pierre. (refus)

Le linguiste constate que, lorsque l'adverbe malheureusement suit la négation, ce double jugement (entre ledit adverbe et l'incise et c'est regrettable) constitue une redondance plutôt inacceptable. Le locuteur naïf focalise intuitivement sur l'adverbe malheureusement en s'interrogeant, tout simplement, sur son opportunité par rapport à celle de l'incise.

3) Tu ne te rappelles pas, naturellement, la première fois que je t'ai embrassé ? (acceptation)

Le linguiste procède à l'analyse des modalités de cette phrase. Il constate que l'exemple dissimule une modalité déclarative, contrairement aux signes de ponctuation ou à l'intonation. Le véritable sens de la phrase est "Je sais (je ne suis pas dupe) que tu ne te rappelles pas la première fois que je t'ai embrassé. " L'ordre des mots (qui est celui de la modalité déclarative) et l'adverbe naturellement cohabitent de manière sémantiquement harmonieuse. Le locuteur naïf, guidé par le sentiment linguistique, saisit le sens déclaratif de la phrase et considère comme conforme à ses propres habitudes énonciatives cette position de l'adverbe.

4) Te rappelles-tu, naturellement, la première fois que je t'ai embrassé ? (refus)

Le linguiste s'appuie sur l'ordre des mots qui, dans ce cas précis, ne peut renvoyer qu'à une modalité interrogative totale (inversion sujet-verbe). Sachant que l'adverbe nafurellement véhicule une valeur de confirmation, il réfutera la phrase à cause de son incohérence sémantico-syntaxique. Le locuteur naïf considère que naturellement est un intrus dans la phrase, dans la mesure où l'on s'attendrait à ce mot éventuellement dans la réponse à la question posée. Il est également à même de reconnaître, toujours intuitivement, une vraie question à l'intérieur de l'exemple.

5) C'est le film que je t'ai parlé.

Le linguiste connaît la propriété syntaxique du verbe parler (dans cet emploi) et est en mesure de la nommer : un emploi prépositionnel, un verbe de second actant, une transitivité indirecte, etc. Par conséquent, il introduit le pronom adéquat, celui qui accompagne ladite propriété : C'es le film dont je t'ai parlé. Le locuteur naïf se contente d'observer, dans le meilleur des cas, que "cela ne se dit pas ". En effet, son savoir intuitif et expérimental de la langue lui procure la capacité de réfuter par élimination non pas le pronom que, mais plus globalement la phrase tout entière comportant ce pronom. D'ailleurs, il peut ne pas connaître l'appartenance de ce mot à la catégorie des pronoms.

Manifestement, le mot "intuition " trouve son utilité au sein de l'analyse linguistique où il fonctionne soit comme une échappatoire interprétative (une solution forcément provisoire), soit comme un ersatz latent d'une explication grammaticale désespérément déficitaire. Or il s'agit précisément dans ce travail de donner à ce concept un contenu théorique, en mettant à nu une structure mentale tacite, livreuse d'un savoir linguistique que nous sommes incapables de formuler 
explicitement. Cependant, nous n'ignorons pas que la chose est obscure, en linguistique comme ailleurs, que l'intuition est souvent trompeuse et qu'il s'agit, avant tout, de démystifier, voire même de démythifier ledit concept dans le cadre de sa réception en sciences du langage.

Revenons donc à l'examen critique des sciences, à l'épistémologie, pour essayer de justifier davantage l'intérêt de cette étude ${ }^{16}$. Les guillaumiens savent que la glossogénie, ou plus précisément l'ontogénie, donne naissance à ce que Guillaume appelle lui-même mécanique intuitionnelle, voire science intuitionnelle (opération constructrice du langage). Il s'agit d'une sorte de science inscrite dans la langue, une pré-science, une avant-science " en face de la science proprement dite, issue de la réflexion conduite, dirigée. " ${ }^{17}$. Guillaume considère que « la connaissance de cette mécanique intuitionnelle est un aboutissant de la science du langage. " ${ }^{18}$.

L'intuition à laquelle nous nous intéressons est effectivement issue de cette infinitude notionnclle qui va de la conceptualisation la plus indéterminée au discernement le plus convaincant, et où le cheminement du raisonnement n'est pas perçu par le locuteur, alors qu'en revanche, la conclusion lui apparaît. Cependant, cette intuition ne fournit pas la définition existentielle du langage, dans la mesure où elle n'en représente qu'une seule composante, à savoir le mentalisme de signifiance et en aucun cas le physisme de représentation. Schématiquement, on obtient l'image suivante :

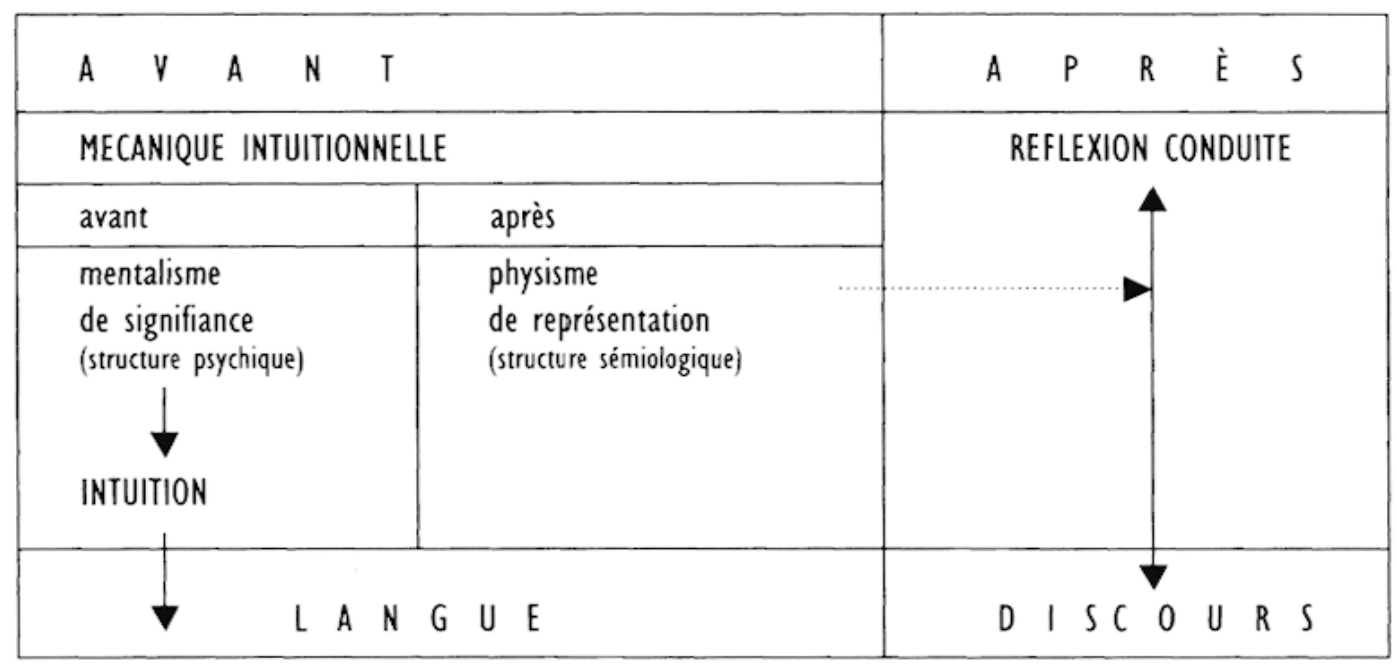

Gustave Guillaume dit ceci à propos de l'intuition : "Nous connaissons la vérité non seulement par la raison, mais encore par le cœur... Et c'est précisément sur ces connaissances du cœur et de l'instinct qu'il faut que la raison s'appuie, et qu'elle y fonde tout son discours. " ${ }^{19}$. C'est ainsi que l'intuition assure le passage que traversent les mots en allant de la langue (domaine appelé en puissance) au discours (domaine appelé en effet). La description de cette opération renforce, une fois de plus, le statut (privilégié) dont bénéficie le mentalisme en linguistique.

16. À titre de parenthèse, un théoricien a avant tout cette délicate mission qui consiste à convaincre avant même de procéder à la vérification (probable) des postulats.

17. Guillaume, 1985, p. 226.

18. A. Boone et A. Joly, 1996, p. 269.

19. Guillaume, 1973, p. 43. 
De son côté, Lucien Tesnière, qui porte aux nues $W$. von Humboldt, parle d'un linguiste "aux intuitions de génie » ${ }^{20}$. Ensuite, il développe un concept qui rappelle étrangement celui $d^{\prime}$ " intuition », mais auquel il réserve le terme de connexion. La connexion est le lien qui unit les mots entre eux et sans lequel il n'y aurait pas de phrase : Alfred parle $=$ Alfred + connexion (intuition) + parle. La connexion ne constitue pas uniquement un mécanisme que le locuteur perçoit intuitivement. La connexion se confond avec l'intuition, plus précisément avec l'intuition énonciative (voir plus loin la définition des différentes intuitions).

En introduisant le concept d'innere Sprachform ("forme intérieure de la langue »), W. von Humboldt renvoie à une compétence mentale qui est antérieure à toute énonciation, et qui se situe du côté de la méthode introspective dont parle précisément Tesnière. Ce mécanisme "prépare " l'énonciation sur le plan psychologique, tandis que la connexion syntaxique permet à l'énonciation de se matérialiser. Il faut savoir que la charpente de la théorie humboldtienne contient trois substantifs verbaux dont le sens est très significatif pour ladite notion : Anschauen («perception »), Denken (" pensée ») et Fühlen (" sentiment»).

$$
\text { * } *
$$

Nous définirons l'intuition en linguistique et ses différents versants de la manière suivante :

L'intuition linguistique désigne la capacité mentale du locuteur à appréhender différemment les structures langagières, avant que n'interviennent les explications analytiques. À l'intérieur de cette notion globalisante résident trois sous-catégories : l'intuition énonciative, l'intuition heuristique et l'intuition analogique.

L'intuition énonciative est une connaissance latente de la langue aboutissant à une construction plus ou moins inconsciente du discours. La plupart du temps, nous avons l'impression que notre réflexion est relativement peu conduite et que nous parlons par une sorte d'inertie mentale. La langue nous " oblige " à dire telle chose plutôt que telle autre.

L'intuition heuristique est une appréhension implicite du système grammatical, une façon de s'approprier les lois inhérentes à la langue sans recourir à la vérification. Très souvent, nous sommes intimement convaincus que telle phrase est grammaticalement inattaquable ou, au contraire, qu'elle est litigieuse, sans pouvoir dire pourquoi. Nous "voyons", intuitivement, que sa structure syntaxique et l'opportunité de son emploi correspondent au génie de la langue.

L'intuition analogique est une compétence que l'on acquiert avec le temps et l'expérience, celle des linguistes, qui permet d'extraire de la masse des exemples des faits particuliers qui intéressent l'analyse linguistique. À la différence du locuteur naïf, le linguiste professionnel possède cette capacité d'identification, d'isolement classificatoire des éléments linguistiques qui le conduit à la reconnaissance du critère de pertinence.

20. Tesnière, 1982, p. 13. 
Voici quelques exemples qui relèvent de différents types de l'intuition linguistique :

(?) Pourriez-vous me consacrer quelques/plusieurs instants? : intuition heuristique. Le locuteur naïf valide la phrase si elle comporte le déterminant quelques, mais il la considère comme douteuse, sans recourir à l'argumentation, si elle comporte le déterminant plusieurs.

(?) Aujourd'hui est morte maman. : intuition heuristique. L'ordre des mots paraît suspect, d'autant plus qu'il rompt avec celui de la célèbre phrase $d^{\prime} A$. Camus : Aujourd'hui, maman est morte.

Ce n'est pas Luc qui m'écrira. : intuition analogique. Le linguiste y reconnaît une négation dissimulée consistant à dire que «Luc ne risque pas de m'écrire ; il n'a pas l'habitude de le faire ", alors que la structure de cette phrase (grâce à la forme négative de l'introducteur c'est...qui) laisse croire qu'il s'agit d'une emphase syntaxique interprétable comme: Ce n'est pas Luc qui m'écrira. C"est plutôt Pierre ou Marie.

Tu veux une fessée? : intuition heuristique. Tout enfant francophone, qui ignore sur le plan proprement intellectuel la notion de modalité phrastique, reconnaît à l'intérieur de cette phrase la modalité injonctive et, par conséquent, ne la confond pas avec la phrase : Tu veux un gâteau?

L'intuition énonciative va de la langue au discours, c'est-à-dire de la finitude à l'infinitude, de ce qui est confirmé à ce qui doit l'être. L'intuition heuristique, à l'inverse, va du discours à la langue, c'est-à-dire de l'infinitude à la finitude, de ce qui doit être confirmé à ce qui l'est déjà.

LANGUE intuition énonciative

DISCOURS

dicibilité en puissance

LANGUE

intuition heuristique

DISCOURS

acceptabilité en effet

À propos de l'intuition linguistique, il existe un autre domaine "vierge " sur lequel ni Guillaume ni Tesnière ne se sont prononcés et que nous nous contenterons d'évoquer sans toutefois l'approfondir. C'est le rapport entre l'intuition et la dichotomie acquisition d'une langue/apprentissage d'une langue. L'acquisition d'une langue, au sens rigoureux, constitue un processus mental inconscient. L'intuition est définie comme un mécanisme mental également inconscient. L'apprentissage d'une langue, en revanche, représente un processus conscient, puisqu'il implique une réflexion sur la langue que l'on apprend et, de surcroît, à l'âge linguistiquement adulte (11-12 ans).

Lorsqu'un locuteur acquiert une langue, au sens rigoureux, il n'acquiert pas un savoir sur cette langue (chez Guillaume, savoir renvoie à réflexion conduite). Il s'approprie le sens en ignorant la nature des formes linguistiques En revanche, l'apprentissage d'une langue procure au locuteur un véritable savoir sur cette langue et sur ses emplois. Le locuteur s'approprie davantage une connaissance des formes qu'une maîtrise du sens. Il en résulte que si le degré d'intuition est plus élevé dans le cas de l'acquisition d'une langue, c'est précisément parce que les deux formes de l'inconscient, celle de l'acquisition et celle de l'intuition, peuvent se superposer. 


\section{CONCLUSION}

Nous terminerons par une conclusion sommaire. Beaucoup reste à faire. Le mentalisme est une véritable infinitude, "l'inévitable credo » (Guillaume). Entre lui et l'intuition, l'esprit aperçoit un rapport de causalité, une immense source d'activités cognitives qui caractérisent la nature humaine. L'un ne va pas sans l'autre : le mentalisme est intuitif, l'intuition est un mécanisme mental. La mission que doit accomplir l'intuition ne consiste pas à "augmenter notre savoir ", mais à améliorer " notre lucidité, la lucidité sans laquelle l'acquisition du savoir serait impossible. » ${ }^{21}$. Après l'intuitionnisme de Bergson en philosophie, la place doit être faite à un intuitionnisme linguistique, qui ne saurait se substituer à une forme de solipsisme.

Il s'agit d'abord d'exploiter la notion d'intuition dans les domaines linguistiques qui ont été évoqués, quitte à augmenter le nombre des définitions proposées en fonction des spécificités épistémologiques que l'on peut adopter. Puis, délimiter une nouvelle fois les niveaux de l'analyse linguistique en les soumettant, un par un, au test intuitionniste : existe-t-il une phonétique intuitive ? L'intuition peut-elle servir la linguistique textuelle? Comment définir le lien entre l'intuition et la notion de continu en linguistique ?...

\section{Bibliographie}

BAJRIC S., 2000. Questions de troduction et d'intentionnalité, dans Style, Visée et Intentionnalité, pp. 77-95, Éditions Le texte et L'Édition, Université de Bourgogne, Dijon.

BAJRIC S., 2003. Mentolisme en linguistique, dans Actes du $2^{\circ}$ colloque sur les études françaises en Croatie. Artresor Naklada, Zagreb, pp. 46-61.

BOONE A. et A. JOLY, 1996, Dictionnaire terminorogique de la systématique du langage, L'Harmattan, Paris.

Ćosic V., 1987. Gustave Guillaume. Psihosistematika: definicija i metoda. dans Strani jezici. 16/1987. n 3-4. pp. 169-175. Zagreb.

ĆOsić V., 1996. Teorija kao sudbina, dans Hrvatsko slovo du 21 juin 1996, p. 6. Zagreb.

De Carvalho P., Quayle N., Rosier L., So JTet O. (sous la direction), 200I, Lo psychoméconique aujourd hui. Actes du $8^{\mathrm{e}}$ Colloque international de psychomécanique du langage. Seyssel. 1997. Honoré Champion, Paris.

DORTIER J.F., 2004. L'homme, cet étrange animal... Éditions Sciences humaines. Auxerre.

FODOR J., 2004 (1983), La modularité de l'esprit, Les Éditions de Minuit, Paris.

GARDES J., 1986, Existe-t-il des niveaux d'acquisition du langage chez l'enfant de deux ò six ans?, dans Cercle linguistique d'Aix-en-Provence. pp. 131-147. Éditions Université de Provence. Aix-en-Provence.

GILSON E., 1969. Linguistique et philosophie. Essa sur les constantes philosophiques du langage. Vrin, Paris.

GUilLAUME G., 1964, Langage et science du langage, Librairie A.G. Nizet, Paris. PUL, Québec.

Guillaume G., 1973. Principes de linguistique théorique. PUL, Québec. Klincksieck. Paris.

GuillaUme G., 1985. Leçons de linguistique, publiées sous la direction de R. Valin. W. Hirtle et A. Joly. 1945-1946. Série C. Tome 6. Grammaire particulière du français et grammaire générale. PUL. Québec, PUL. Lille.

LEEMAN D., 2002, La phrose complexe, De Boeck. Duculot, Bruxelles.

PUECH C. et SAVATOVSKY D., 1982. Structuralisme et/ou Mentalisme? Gustave Guilloume, LINX $n^{\circ} 6$, PP. 121 - I54. Paris.

ROBiNS R.H., 1976 (1967), Brève histoire de lo linguistique, Éditions du Seuil, Paris.

TESNIERE L., 1982 (1959), Éléments de syntaxe structurule. Klincksieck. Paris.

WITTGENSTEIN L.. 1963. Tractotus logico-philosophicus, Logisch-philosophische Abhandlung. Suhrkamp. Frankfurt am Main.

YAGUELlo M.. 1988. Cotalogue des idées reçues sur la langue. Point virgule. Paris.

21. Guillaume, 1973, p. 44. 